\title{
Enhanced MIN-6 beta cell survival and function on a nitric oxide-releasing peptide amphiphile nanomatrix
}

This article was published in the following Dove Press journal:

International Journal of Nanomedicine

5 May 2014

Number of times this article has been viewed

\author{
Dong-Jin Lim ${ }^{1, *}$ \\ Adinarayana Andukuri',* \\ Jeremy B Vines ${ }^{1,2}$ \\ Shibli M Rahman' \\ Patrick TJ Hwang' \\ Jeonga $\mathrm{Kim}^{3}$ \\ Anath Shalev 4,6 \\ John A Corbett ${ }^{5}$ \\ Ho-Wook Jun ${ }^{1,6}$ \\ 'Department of Biomedical \\ Engineering, University of Alabama \\ at Birmingham, ${ }^{2}$ Nutech Medical \\ Inc, ${ }^{3}$ Department of Medicine, \\ Division of Endocrinology, Diabetes \\ and Metabolism, ${ }^{4}$ Department of \\ Medicine, University of Alabama \\ at Birmingham, Birmingham, AL, \\ ${ }^{5}$ Department of Biochemistry, Medical \\ College of Wisconsin, Milwaukee, WI, \\ ${ }^{6}$ Comprehensive Diabetes Center, \\ University of Alabama at Birmingham, \\ Birmingham, AL, USA \\ *These authors contributed equally \\ to this work
}

\begin{abstract}
Innovative biomaterial strategies are required to improve islet cell retention, viability, and functionality, and thereby obtain clinically successful outcomes from pancreatic islet cell transplantation. To address this need, we have developed a peptide amphiphile-based nanomatrix that incorporates multifunctional bioactive cues and sustained release of nitric oxide. The goal of this study was to evaluate the effect of this peptide amphiphile nanomatrix on the viability and functionality of MIN-6 islet cells. Additionally, this study provides insight into the role of nitric oxide in islet cell biology, given that conventional nitric oxide donors are unable to release nitric oxide in a controlled, sustained manner, leading to ambiguous results. It was hypothesized that controlled nitric oxide release in synergy with multifunctional bioactive cues would promote islet cell viability and functionality. Nitric oxide-releasing peptide amphiphile nanomatrices within the range of $16.25 \mu \mathrm{mol}$ to $130 \mu \mathrm{mol}$ were used to analyze MIN-6 cell behavior. Both $32.5 \mu \mathrm{mol}$ and $65 \mu \mathrm{mol}$ peptide amphiphiles showed improved MIN-6 functionality in response to glucose over a 7-day time period, and the elevated functionality was correlated with both PDX-1 and insulin gene expression. Our results demonstrate that nitric oxide has a beneficial effect on MIN-6 cells in a concentration-dependent manner.
\end{abstract}

Keywords: nitric oxide, peptide amphiphile, biomimetic, diabetes, beta cells

\section{Introduction}

Pancreatic islet transplantation is a promising new mode of treatment for type 1 diabetes. ${ }^{1}$ Successful outcomes from pancreatic islet transplantation could lead to resolution of the complications associated with type 1 diabetes that lead to mortality and morbidity. ${ }^{2,3}$ However, the potential advantages and clinical successes of pancreatic islet transplantation are dependent on development of innovative biomaterial strategies. These strategies are required to fulfill the critical needs for success of pancreatic islet transplantation by improving islet cell retention, viability, and functionality. In response to these needs, peptide amphiphile (PA)-based biomaterials that closely mimic the islet cell microenvironment and extracellular matrix proteins have been garnering increasing attention. ${ }^{4-7}$ Peptide amphiphiles are molecules that consist of a hydrophilic, functional peptide sequence coupled to a hydrophobic alkyl tail. ${ }^{8-10}$ The amphiphilic nature of the molecule drives the self-assembly of PAs into a three-dimensional nanomatrix, and inclusion of multifunctional bioactive cues in the functional peptide sequence regulate islet cell behavior.

For this study, we developed a peptide amphiphile nanomatrix that incorporates a laminin-derived cell adhesive ligand (Tyr-Ile-Gly-Ser-Arg), a matrix metalloprotease-2 degradable site (Gly-Thr-Ala-Gly-Leu-Ile-Gly-Gln), ${ }^{9}$ and a nitric oxide (NO) 
donor (Lys-Lys-Lys-Lys-Lys) $)^{11-14}$ to provide cells with the requisite bioactive cues. Laminin is a major component of the islet cell extracellular matrix, and laminin-derived sequences have been previously investigated, with favorable outcomes. ${ }^{15}$ Moreover, our previous study showed that the laminin cell adhesive ligand, Tyr-Ile-Gly-Ser-Arg, enhances survival and function of MIN-6 beta cells, and the matrix metalloprotease-2 degradable site allows for cell-mediated degradation of the nanomatrix, thereby promoting cell migration and production of native extracellular matrix..$^{9,16,17}$ Tunable NO release from this nanomatrix has been shown to occur in a controlled biphasic manner, with a burst release followed by sustained release over a 30-day period. ${ }^{11,12}$ Previous studies of this PA nanomatrix have shown great potential for applications in cardiovascular implant devices such as stents and vascular grafts. ${ }^{11,13}$ The nanomatrix can be self-assembled into two-dimensional coatings or three-dimensional nanomatrix gels. ${ }^{18-20}$ It has been shown to promote endothelial cell adhesion and proliferation ${ }^{11,12}$ as well as endothelial progenitor cell adhesion and differentiation. ${ }^{14}$ Therefore, the nanomatrix has the potential to stimulate and support angiogenesis.

Thus, the goal of this work was to study the effect of the NO-releasing PA nanomatrix on the viability and functionality of MIN-6 beta cells, which have similar physiologic features to islets. ${ }^{21}$ The PA nanomatrix is composed of two PAs, ie, one PA containing the laminin-derived cell adhesive ligand (YIGSR) linked to the matrix metalloprotease-2 degradable site (GTAGLIGQ) to form PA-YIGSR, and the second PA containing a polylysine NO donor linked to the matrix metalloprotease-2 degradable site to form PA-KKKKK. PAYIGSR and PA-KKKKK were mixed in a 9:1 molar ratio based on previous studies, ${ }^{11-14}$ and reacted with pure NO to form NO-releasing PA, called PA-YK-NO. Self-assembly of PA-YK-NO into uniform nanofibers and controlled, biphasic NO release have been previously demonstrated. ${ }^{11}$

However, the role of NO in islet cell biology remains a source of ambiguity and controversy. The lack of NO donors that can release NO in a controlled, sustained manner can lead to results that cannot be reliably interpreted. ${ }^{22}$ Some studies suggest a detrimental role, where NO mediates the inflammatory reaction in response to engrafted islet cells. ${ }^{23-27}$ This has been evidenced by elevated expression of interleukin-1 $\beta$ and inducible nitric oxide synthase in regions of pancreatic islet allotransplantation. ${ }^{28,29}$

However, some studies suggest that NO stimulates insulin production and cell viability. ${ }^{30-33}$ This would suggest that the role of NO in islet cell biology may be concentration-dependent. Furthermore, conventional NO donors do not provide the islet cells with other bioactive cues that promote cell viability and functionality. This study therefore provides the added opportunity of studying the effect of controlled NO release on islet cells. We hypothesized that controlled NO release in synergy with multifunctional bioactive cues incorporated into the PA nanomatrix can improve islet cell viability and functionality.

\section{Materials and methods}

Preparation of peptide amphiphiles

Two peptide amphiphiles, ie, PA-YIGSR $\left[\mathrm{CH}_{3}\left(\mathrm{CH}_{2}\right)_{14} \mathrm{CONH}-\right.$ GTAGLIGQ-YIGSR] and PA-KKKKK $\left[\mathrm{CH}_{3}\left(\mathrm{CH}_{2}\right)_{14} \mathrm{CONH}-\right.$ GTAGLIGQ-KKKKK] were prepared using the Fmoc chemistry in the Advanced Chemtech Apex 396 peptide synthesizer (AAPPTec, Louisville, KY, USA) and subsequently were alkylated at the N-termini with palmitic acid by a manual coupling reaction for 24 hours at room temperature. ${ }^{18,20}$ To alkylate with palmitic acid, a mixture of o-benzotriazole-N,N,N,N'-tetramethyluronium hexafluorophosphate, diisopropylethylamine, and dimethylformamide was used, and cleavage and deprotection were achieved using a mixture of trifluoroacetic acid, deionized water, triisopropylsilane, and anisole (40:1:1:1) for 3 hours at room temperature. The peptide amphiphiles precipitated in cold ether were lyophilized and characterized by matrix-assisted laser desorption ionization time of flight mass spectrometry.

\section{Preparation of NO-releasing PA-YK-NO nanomatrices}

As documented in previous work, a mixture of PA-YIGSR and PA-KKKKK at a 9:1 molar ratio was reacted with NO gas to generate PA-YK-NO. ${ }^{11}$ In order to make NO-releasing PA nanomatrices, $120 \mu \mathrm{L}$ of a $0.1 \%$ wt stock PA-YK-NO solution (pH 7.4) was prepared and dispensed into 48-well tissue culture plates (BD Biosciences, San Jose, CA, USA) with serial dilutions to make different PA-YK-NOs, ie, $130 \mu \mathrm{mol}$ PA-YK-NO, $65 \mu \mathrm{mol}$ PA-YK-NO, $32.5 \mu \mathrm{mol}$ PA-YK-NO, and $16.25 \mu \mathrm{mol}$ PA-YK-NO.

\section{MIN-6 cell culture on PA-YK-NO nanomatrices}

MIN-6 cells were received from Donald F Steiner (Howard Hughes Medical Institute, Department of Biochemistry, University of Chicago, Chicago, IL, USA) with the permission of Jun-Ichi Miyazaki (Division of Stem Cell Regulation Research [G6], Osaka University Graduate School of Medicine, Osaka, Japan). MIN-6 cells were routinely expanded in $75 \mathrm{~cm}^{2}$ tissue culture-treated flasks at $37^{\circ} \mathrm{C}$ in an atmosphere 
of $95 \%$ air and $5 \% \mathrm{CO}_{2}{ }^{7}$ Dulbecco's modified Eagle's medium containing $25 \mathrm{mmol} / \mathrm{L}$ glucose supplemented with $15 \%$ fetal bovine serum (Atlanta Biologicals, Lawrenceville, GA, USA), 100 units/mL penicillin (Invitrogen, Carlsbad, CA, USA), $100 \mu \mathrm{g} / \mathrm{mL}$ streptomycin (Invitrogen), $100 \mu \mathrm{g} / \mathrm{mL}$ L-glutamine (Invitrogen), and $5 \mu \mathrm{l} / \mathrm{L} \beta$-mercaptoethanol (Gibco, Carlsbad, CA, USA) was used to culture the MIN-6 cells. The cells were seeded at a density of 42,000 per $\mathrm{cm}^{2}$ and incubated for 3 and 7 days, with plates from 7-day cultivations refilled with fresh medium at 3 days.

\section{Glucose-stimulated insulin secretion assay}

To assess MIN-6 functionality in response to glucose, glucosestimulated insulin secretions at 3 and 7 days were performed for 2 hours in each condition with $22 \mathrm{mmol} / \mathrm{L}$ KrebsRinger bicarbonate buffer solutions $(25 \mathrm{mmol} / \mathrm{L}$ HEPES, $115 \mathrm{mmol} / \mathrm{L} \mathrm{NaCl}, 24 \mathrm{mmol} / \mathrm{L} \mathrm{NaHCO}_{3}, 5 \mathrm{mmol} / \mathrm{L} \mathrm{KCl}$, $1 \mathrm{mmol} / \mathrm{L} \mathrm{MgCl}_{2}, 2.5 \mathrm{mmol} / \mathrm{L} \mathrm{CaCl}_{2}, 0.1 \%$ bovine serum albumin, and $22 \mathrm{mmol} / \mathrm{L}$ D-glucose, $\mathrm{pH}$ 7.4). Using an Ultra Sensitive Rat Insulin ELISA kit (Crystal Chem Inc, Downers Grove, IL, USA), the collected samples were quantified and normalized by DNA content to obtain the secreted insulin values. The DNA content was quantified using a fluorometric PicoGreen DNA kit (Molecular Probes, Eugene, OR, USA) with a microplate fluorescent reader (Synergy HT, Bio-Tek Instruments, Winooski, VT, USA).

\section{Caspase $3 / 7$ activity in MIN-6 cells cultured on PA-YK-NO nanomatrices}

The caspase activity of MIN-6 cells cultured on four different PA-YK-NOs was assayed using the Caspase-Glo 3/7 assay kit (Promega, Madison, WI, USA) to assess cellular apoptosis. Briefly, each PA was coated onto a white, opaque 96-well plate, and MIN-6 cells (42,000 per $\left.\mathrm{cm}^{2}\right)$ were cultured for 3 and 7 days. At each time point, the cells were washed with phosphate-buffered saline, and $100 \mu \mathrm{L}$ of fresh medium and $100 \mu \mathrm{L}$ of the buffered Caspase-Glo substrate were added. After 3 hours, the amount of caspase activity was evaluated by luminescence (Synergy HT, Bio-Tek Instruments).

\section{Evaluation of cellular behavior on PA-YK-NO nanomatrices}

MIN-6 cells were cultured on four different PA-YK-NOs for 3 and 7 days. At each time point, the media was aspirated, and the cells were rinsed with phosphate-buffered saline.
MIN-6 cell viability for each condition was assessed using the Live/Dead Assay Kit (Molecular Probes Inc) consisting of calcein AM and ethidium homodimer-1.

\section{Quantitative real-time polymerase chain reaction}

Using Trizol regent (Invitrogen), total RNA was collected for all samples in each condition at days 3 and 7. For measurement of ins 1 and PDX-1 gene expression, all samples were incubated for 2 hours in the presence of glucose $(22 \mathrm{mM})$ and then subjected to RNA extraction. The isolated samples were pelleted, dried in ethanol, and resuspended in nuclease-free water. DNase treatments were carried out (TURBO DNase, Ambion, Austin, TX, USA) before performing quantitative real-time polymerase chain reaction. The RNA concentration of each sample was measured with an ND-1000 UV spectrophotometer (Nanodrop, Wilmington, DE, USA), and $1 \mu \mathrm{g}$ of isolated RNA was then reverse-transcribed into cDNA using a iScript cDNA Synthesis Kit (Bio-Rad, Hercules, CA, USA) according to the manufacturer's instructions. All quantitative real-time polymerase chain reactions were carried out using a Prism 7000 Sequence Detection System (Applied Biosystems, Foster City, CA, USA) with the iQ SYBR Green Supermix (Bio-Rad). The primers used in this study are described in Table 1, and 18S rRNA was used as the internal standard (18 s RNA forward: 5'-AGT CCC TGC CCT TTG TAC ACA-3', reverse: 5'-GAT CCG AGG GCC TCA CTA AAC-3'). The measured values were normalized to the internal standard and calculated using the $2^{-\Delta \Delta \mathrm{Ct}}$ method. After 18S rRNA normalization, gene expression levels were presented as the fold ratio relative to those of the control group (tissue culture plate).

\section{Statistical analysis}

All experiments were performed independently four times, and the normalized values for glucose-stimulated insulin secretion were expressed as the mean \pm standard deviation. One-way analysis of variance was used to examine for

Table I Primer sequences for real-time polymerase chain reaction

\begin{tabular}{cll}
\hline Primer & Sequence $\left(5^{\prime}-\mathbf{3}^{\prime}\right)$ & $\begin{array}{l}\text { GenBank } \\
\text { identification }\end{array}$ \\
\hline $\begin{array}{c}\text { Insulin (ins I) } \\
\text { Forward }\end{array}$ & GAA GTG GAG GAC CCA CAA GTG & NM_008386 \\
$\begin{array}{l}\text { Reverse } \\
\text { PDX-I }\end{array}$ & CTG AAG GTC CCC GGG GCT & \\
Forward & CGC GTC CAG CTC CCT TT & \\
Reverse & CCA CGC GTG AGC TTT GGT & \\
\hline
\end{tabular}


statistical significance. Tukey's multiple comparison tests were used to determine significant differences between pairs. The statistical analysis was performed using Statistical Package for the Social Sciences version 15 software (SPSS Inc, Chicago, IL, USA). $P \leq 0.05$ was considered to be statistically significant.

\section{Results and discussion Evaluation of cellular behavior on the four different PA-YK-NOs}

Four different PA-YK-NOs were used for this study, ie, $130 \mu \mathrm{mol}, 65 \mu \mathrm{mol}, 32.5 \mu \mathrm{mol}$, and $16.25 \mu \mathrm{mol}$ (Figure 1). A PA-YK-NO was composed of two PAs, ie, an extracellular matrix mimetic PA and a NO-releasing PA combined in a 9:1 molar ratio. The extracellular matrix mimetic PA, referred to as PA-YIGSR, contained a cell-adhesive sequence of TyrIle-Gly-Ser-Arg (YIGSR), which has been shown to improve MIN-6 cell survival as well as function. Similarly, the NOreleasing PA had a NO-donating sequence Lys-Lys-Lys-LysLys (KKKKK). Dispensing different concentrations of the PA-YK-NO solution, four different PA-YK-NO nanomatrices were created and used to evaluate MIN-6 cellular behavior in response to NO. All PA-YK-NOs were characterized and confirmed to release NO in a physiologically acceptable concentration over one month. MIN-6 cells were attached and grown with similar cellular behavior in each PA-YKNO nanomatrix (Table 2). Although the average number of cells was slightly greater in the control group at 7 days relative to the PA-YK-NO substrate groups, all PA-YK-NO substrates showed similar growth behavior, with comparable cell viability and cell concentrations. MIN-6 cell viability on the four different PA-YK-NOs was similar, indicating that all the PA-YK-NOs were biocompatible (Figure 2).

\section{Evaluation of functional behavior on the four different PA-YK-NO nanomatrices}

Despite the negative effect on beta cells, recent studies indicate that physiologically acceptable NO exhibits positive effects as well. ${ }^{22,34}$ Enhancing several pathways involved in insulin gene expression, exogenous $\mathrm{NO}$ has been shown to stimulate insulin secretion. When cultured on PA-YK-NO nanomatrices, glucose-stimulated insulin secretion responses ( $22 \mathrm{mM}$ ) for both 3 and 7 days of cultivation were either better or at the same level as the control, indicating that $\mathrm{NO}$ in low concentrations may have a beneficial effect on the functionality of MIN-6 cells. Excluding the highest NO dose, ie, $130 \mu \mathrm{mol}$ PA-YK-NO, all NO-releasing PA nanomatrices had beneficial effects on MIN-6 functionality. Additionally, on $65 \mu \mathrm{mol}$ PA-YK-NO, MIN-6 cells demonstrated the highest level of insulin secretion response, with $32.5 \mu \mathrm{mol}$ PA-YKNO having the second best effect over 7 days (Figure 3). At 3 days, MIN-6 cells showed significantly improved insulin functionality on three of the four NO-releasing PA nanomatrices, whereas $16.25 \mu \mathrm{mol}$ of PA-YK-NO did not show an initial improved response to glucose. This demonstrates that the initial release of NO by the majority of the PA-YK-NO nanomatrices stimulates MIN-6 cells to respond better to glucose, and indicates that the sustained low NO concen-

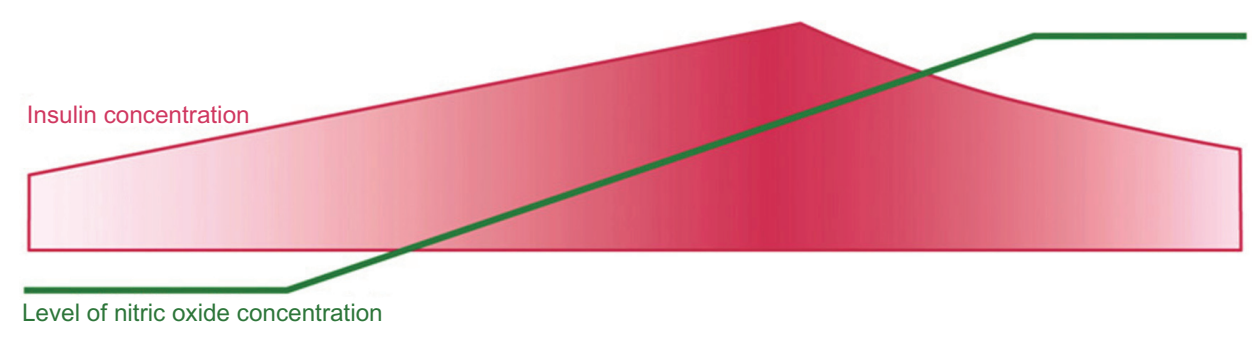

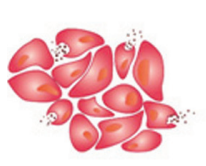

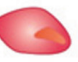

MIN6 $\beta$ cell
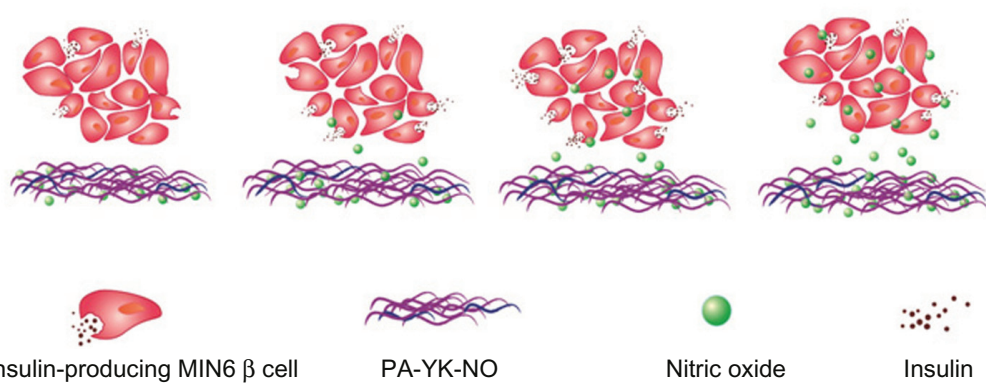

Insulin-producing MIN6 $\beta$ cell

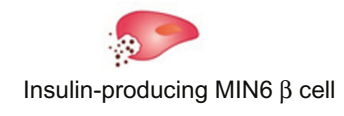

Figure I General schematic summary of the study.

Abbreviation: PA-YK-NO, nitric oxide-releasing peptide amphiphile nanomatrix. 
Table 2 Characterization of MIN-6 cell growth when cultured on different PA-YK-NO nanomatrices

\begin{tabular}{|c|c|c|c|c|c|}
\hline & \multicolumn{2}{|l|}{ Viability } & \multicolumn{2}{|c|}{ Average number of cells $\left(\times 10^{4}\right)$} & \multirow{2}{*}{$\begin{array}{l}\text { Specific growth } \\
\text { rate }\left(\mu_{g, 7 \text { days }}\right)\end{array}$} \\
\hline & 3 days & 7 days & 3 days & 7 days & \\
\hline Control (TCP) & $95.24 \pm 0.42$ & $91.53 \pm 0.37$ & $4.15 \pm 0.16$ & $20.67 \pm 2.00$ & $0.282 \pm 0.003$ \\
\hline I6.25 $\mu \mathrm{mol}$ PA-YK-NO & $96.23 \pm 0.57$ & $90.57 \pm 0.48$ & $5.46 \pm 0.56$ & $|7.3| \pm 2.67$ & $0.260 \pm 0.016$ \\
\hline $32.5 \mu \mathrm{mol}$ PA-YK-NO & $94.16 \pm 3.56$ & $90.33 \pm 0.42$ & $5.27 \pm 0.66$ & $16.47 \pm 2.68$ & $0.241 \pm 0.014$ \\
\hline $65 \mu \mathrm{mol}$ PA-YK-NO & $96.33 \pm 0.56$ & $90.52 \pm 0.22$ & $5.47 \pm 0.71$ & $17.09 \pm 3.64$ & $0.218 \pm 0.026$ \\
\hline I $30 \mu \mathrm{mol}$ PA-YK-NO & $96.20 \pm 0.43$ & $91.20 \pm 0.58$ & $5.60 \pm 0.73$ & $19.64 \pm 1.27$ & $0.261 \pm 0.008$ \\
\hline
\end{tabular}

Abbreviations: PA-YK-NO, nitric oxide-releasing peptide amphiphile nanomatrix; TCP, tissue culture plate.

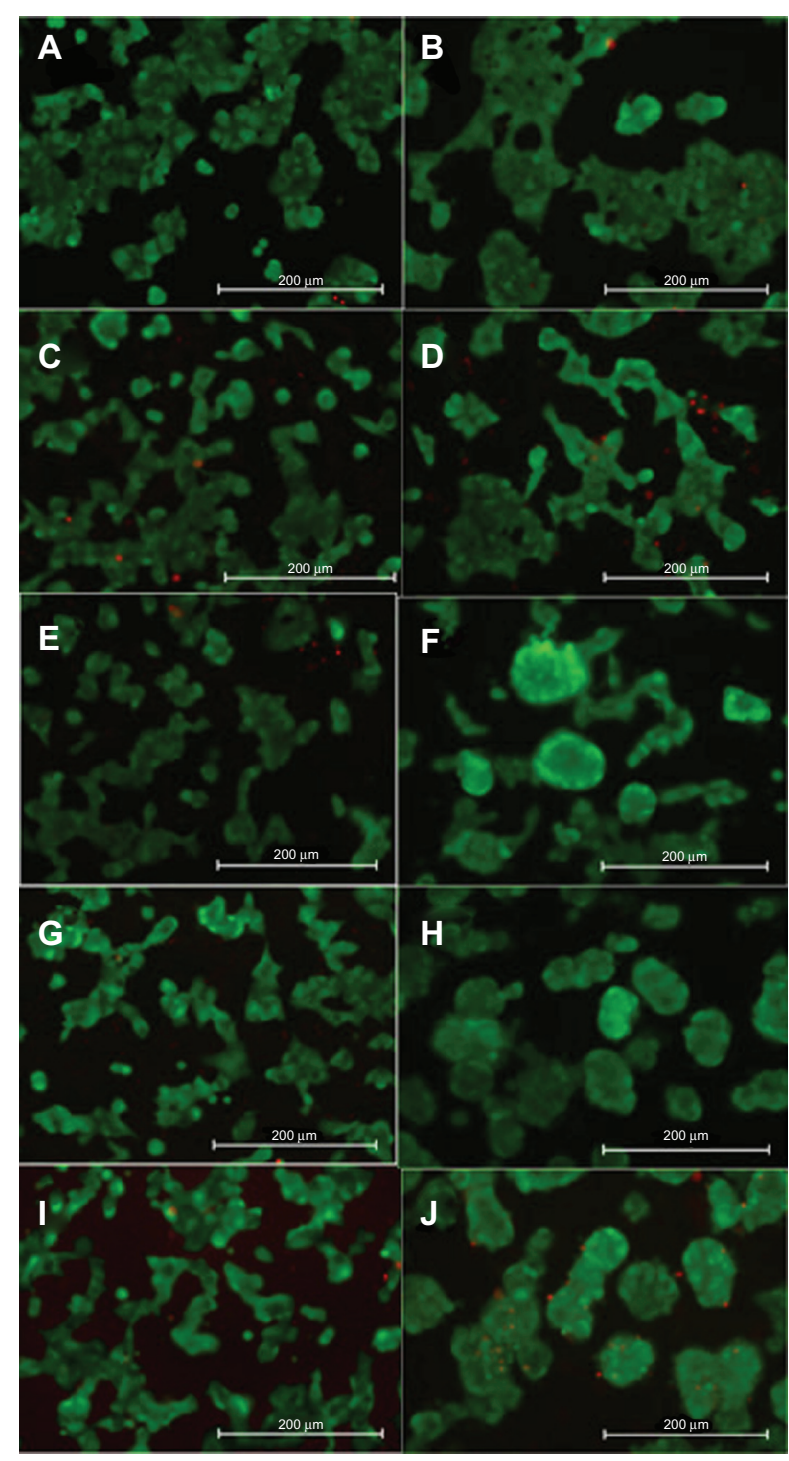

Figure 2 Representative Live/Dead assay images.

Notes: (A) 3 days cultivation of MIN-6 cells on a TCP, (B) 7 days cultivation of MIN-6 cells on a TCP, (C) 3 days cultivation of MIN-6 cells on a $16.25 \mu \mathrm{mol}$ PA-YK-NO nanomatrix, (D) 7 days cultivation of MIN-6 cells on a $16.25 \mu \mathrm{mol}$ PA-YK-NO nanomatrix, (E) 3 days cultivation of MIN-6 cells on a $32.5 \mu \mathrm{mol}$ PA-YK-NO nanomatrix, (F) 7 days cultivation of MIN-6 cells on a $32.5 \mu \mathrm{mol}$ PA-YK-NO nanomatrix, (G) 3 days cultivation of MIN-6 cells on a $65 \mu \mathrm{mol}$ PAYK-NO nanomatrix, (H) 7 days cultivation of MIN-6 cells on a $65 \mu \mathrm{mol}$ PA-YKNO nanomatrix, (I) 3 days cultivation of MIN-6 cells on a $130 \mu \mathrm{mol}$ PA-YK-NO nanomatrix, and (J) 7 days cultivation of MIN-6 cells on a $130 \mu \mathrm{mol}$ PA-YK-NO nanomatrix. All scale bars indicate $200 \mu \mathrm{m}$.

Abbreviations: PA-YK-NO, nitric oxide-releasing peptide amphiphile nanomatrix; TCP, tissue culture plate. trations have a positive effect. Increasing exposure to $\mathrm{NO}$ over time allowed MIN-6 cells to respond to a sustained NO dose. PA-YK-NO at $16.25 \mu \mathrm{mol}$ showed an increase in insulin secretion at 7 days, whereas the $130 \mu \mathrm{mol}$ PA-YKNO did not show any positive effect on MIN-6 functionality. Overall, both $32.565 \mu \mathrm{mol}$ and $65 \mu \mathrm{mol}$ of PA-YK-NO had improved functionality over time, demonstrating that the middle range of PA-YK-NO has the best results regarding MIN-6 functionality.

\section{Quantitative real-time polymerase chain reaction gene expression analysis}

Previous studies indicate that a stimulatory effect of exogenous NO relates to the gene transcription factor PDX-1, which regulates insulin gene expression. Campbell et al found that PDX-1 upregulated by NO improved insulin gene expression, concluding that $\mathrm{NO}$ at low levels could play a regulatory role on insulin secretion in MIN-6 cells. ${ }^{33}$ At 3 days, relatively higher amounts of PA-YK-NO $(130 \mu \mathrm{mol}$ PA-YK-NO and $65 \mu \mathrm{mol}$ PA-YK-NO) showed increased PDX-1 gene expression when compared with the control and $16.25 \mu \mathrm{mol}$ of PA-YK-NO (Figure 4). This upregulation of insulin genes in the higher PA-YK-NOs at 3 days indicates that an initial sustained release of NO by the PA-YK-NOs could have short-term beneficial effects for MIN-6 cells (Figure 5). After 7 days, the $16.25 \mu \mathrm{mol}$ and $32.5 \mu \mathrm{mol}$ PA-YK-NO nanomatrices showed improved PDX-1 gene expression, resulting in a significant increase in insulin gene expression (Figures 6 and 7). These results demonstrate that the beneficial functional role of NO exerted by the biomimetic NO-releasing PAs resulted from upregulation of the PDX-1 gene, which corresponded to the increase in insulin gene expression.

Seven-day observation of the PDX-1 gene and insulin gene expression showed a shift from upregulation at the higher dose of PA-YK-NOs to upregulation at the lower dose of PAYK-NOs. The expression pattern for PDX-1 and the insulin gene explained the functional behavior of the MIN- 6 cells on the biomimetic NO-releasing PA nanomatrices. While being 


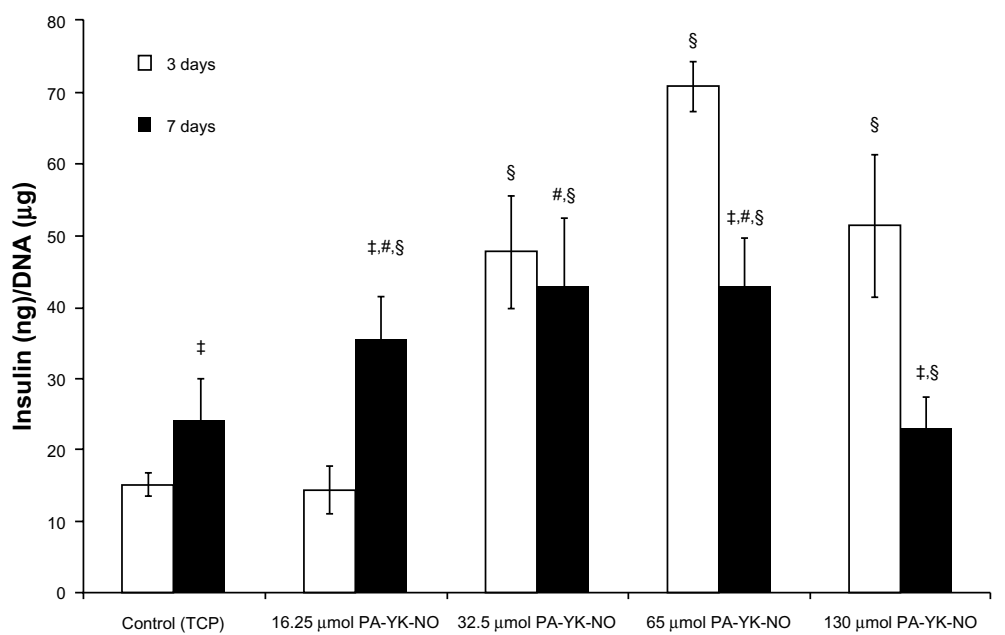

Figure 3 Glucose-stimulated insulin secretion with Krebs-Ringer bicarbonate buffer (22 mmol/L glucose) of MIN-6 cells on different PA-YK-NO nanomatrices.

Notes: At 3 and 7 days, MIN-6 cells were subject to glucose insulin secretion assay for 2 hours. Results are expressed as secreted insulin values per DNA. Error bars represent the mean \pm standard deviation for $n=12$. ${ }^{\ddagger} P<0.05$ compared with secreted value on day 3 in each condition; $\$ P<0.05$ compared with day 3 control; ${ }^{\sharp} P<0.05$ compared with day 7 control after 7 days of cultivation.

Abbreviations: PA-YK-NO, nitric oxide-releasing peptide amphiphile nanomatrix; TCP, tissue culture plate.

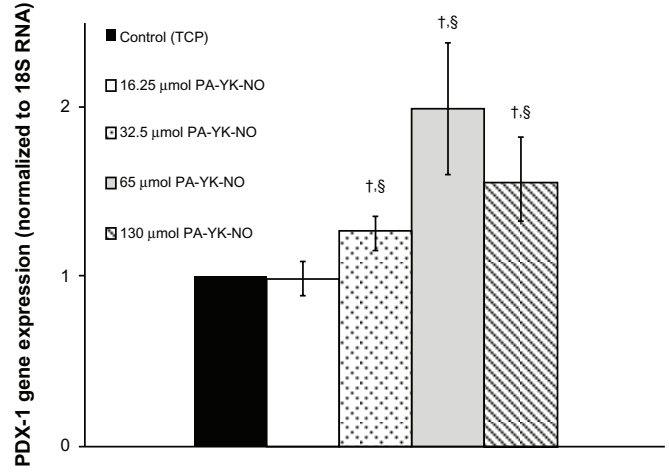

Figure 4 Gene expression profile for PDX-I over 3 days.

Notes: Each value is expressed as the mean \pm standard deviation relative to TCP (black bar). Significant gene expression is found compared with ${ }^{\S} \mathrm{TCP}$ control and $\dagger$ i $6.25 \mu$ mol PA-YK-NO $(P<0.05, \mathrm{n}=12)$.

Abbreviations: PA-YK-NO, nitric oxide-releasing peptide amphiphile nanomatrix; $\mathrm{TCP}$, tissue culture plate.

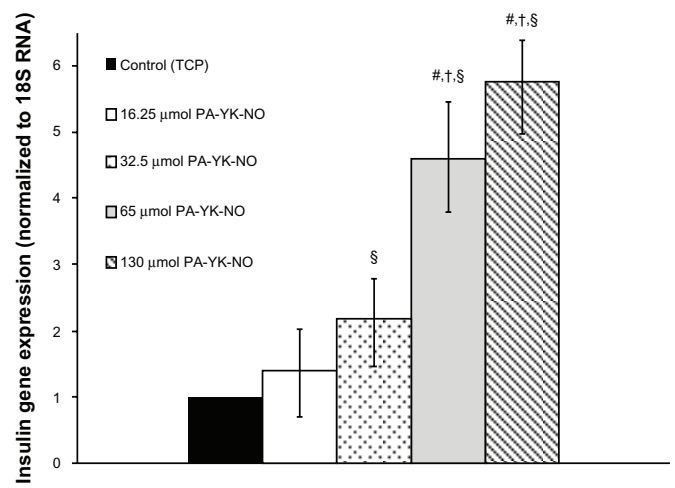

Figure 5 Gene expression profile for insulin (ins I) over 3 days.

Notes: Each value is expressed as the mean \pm standard deviation relative to TCP (black bar). Significant gene expression was found compared with ${ }^{\S} \mathrm{TCP}$ control, ${ }^{\dagger} 6.25 \mu \mathrm{mol}$ PA-YK-NO, and \#32.5 $\mu$ mol PA-YK-NO $(P<0.05, \mathrm{n}=12)$.

Abbreviations: PA-YK-NO, nitric oxide-releasing peptide amphiphile nanomatrix; $\mathrm{TCP}$, tissue culture plate.

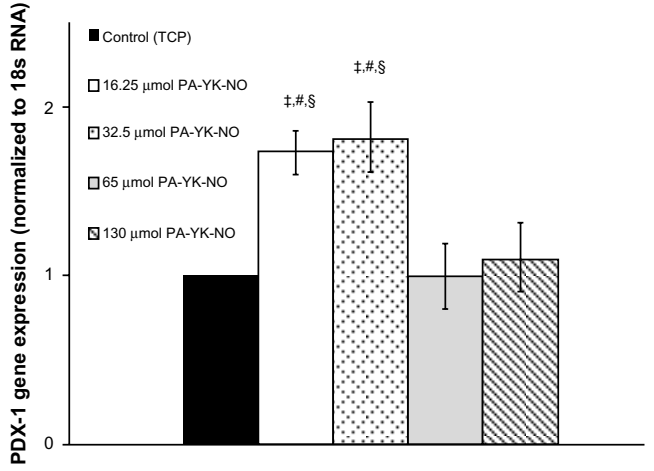

Figure 6 Gene expression profile for PDX-I over 7 days.

Notes: Each value is expressed as the mean \pm standard deviation relative to TCP (black bar). Significant gene expression was found compared with ${ }^{\S} \mathrm{TCP}$ control, ${ }^{\ddagger} 65 \mu \mathrm{mol}$ PA-YK-NO, and ${ }^{\# 1} 30 \mu \mathrm{mol}$ PA-YK-NO $(P<0.05, \mathrm{n}=12)$.

Abbreviations: PA-YK-NO, nitric oxide-releasing peptide amphiphile nanomatrix; TCP, tissue culture plate.

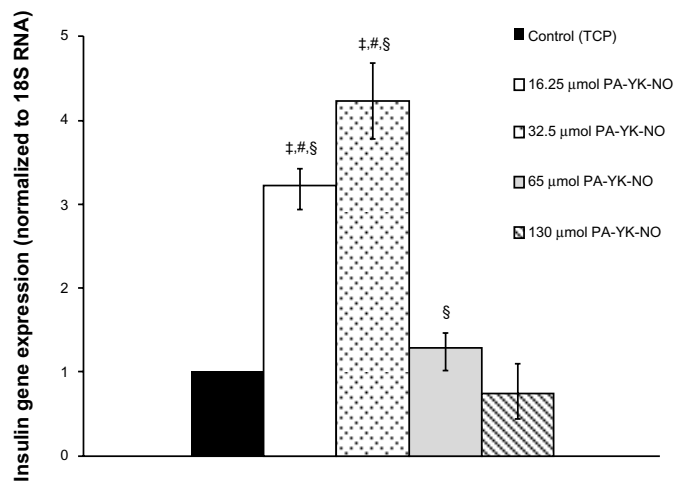

Figure 7 Gene expression profile for insulin (insI) over 7 days.

Notes: Each value is expressed as the mean \pm standard deviation relative to TCP (black bar). Significant gene expression was found compared with ${ }^{\circledR} \mathrm{TCP}$ control, ${ }^{\ddagger} 65 \mu \mathrm{mol}$ PA-YK-NO, and ${ }^{\# 1} 30 \mu \mathrm{mol}$ PA-YK-NO $(P<0.05, \mathrm{n}=12)$.

Abbreviations: PA-YK-NO, nitric oxide-releasing peptide amphiphile nanomatrix; $\mathrm{TCP}$, tissue culture plate. 


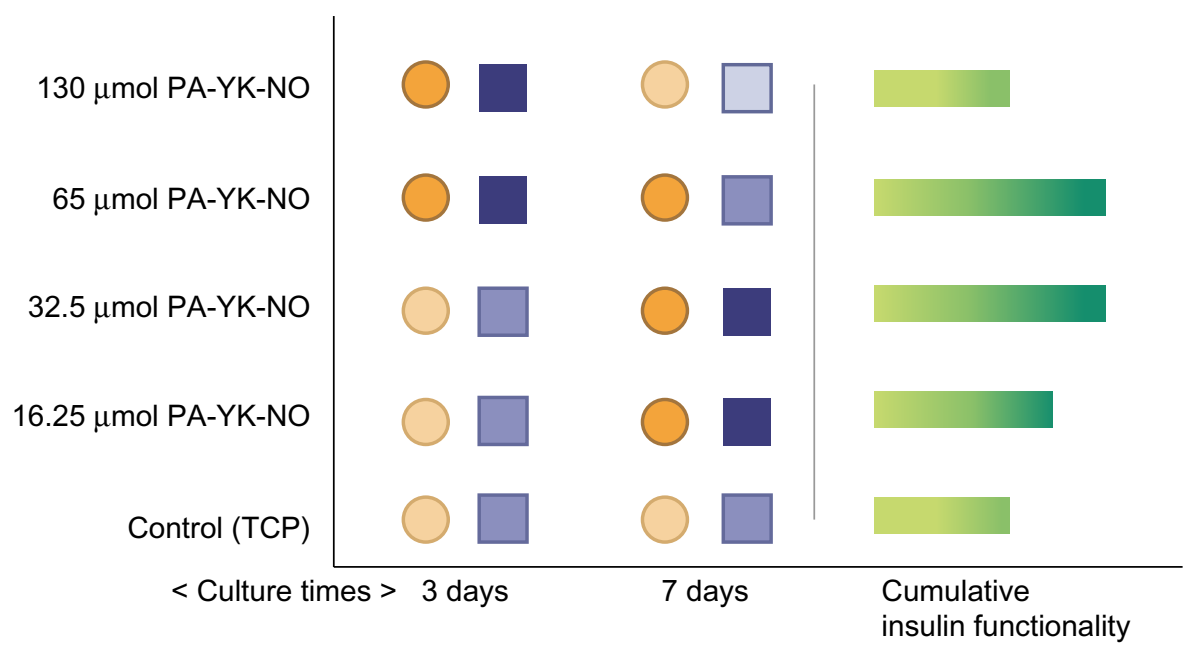

PDX-1 gene

INS-1 gene

Insulin functionality

Figure 8 Schematic summary of the beneficial role of nitric oxide released by the peptide amphiphile nanomatrix on MIN-6 beta cell functionality.

Notes: It shows that the biomimetic nitric oxide-releasing nanomatrix releases nitric oxide continuously within a physiologically acceptable concentration, leading to an improved response to glucose in MIN-6 beta cells.

Abbreviations: PA-YK-NO, nitric oxide-releasing peptide amphiphile nanomatrix; TCP, tissue culture plate.

less responsive to a relatively higher dose of PA-YK-NO (130 $\mu \mathrm{mol})$ over a prolonged period of time, MIN-6 cells cultured on $32.5 \mu \mathrm{mol}$ and $65 \mu \mathrm{mol}$ PA-YK-NO over a longer period of time could have beneficial effects in terms of insulinrelated gene expression as well as functionality.
Overall, this study demonstrated enhanced functionality of MIN-6 beta cells in response to NO delivered by an NOreleasing peptide amphiphile (PA) molecule that mimics the beneficial cellular microenvironment conducive to successful islet transplantation. Our findings indicate that PA-YK-NO

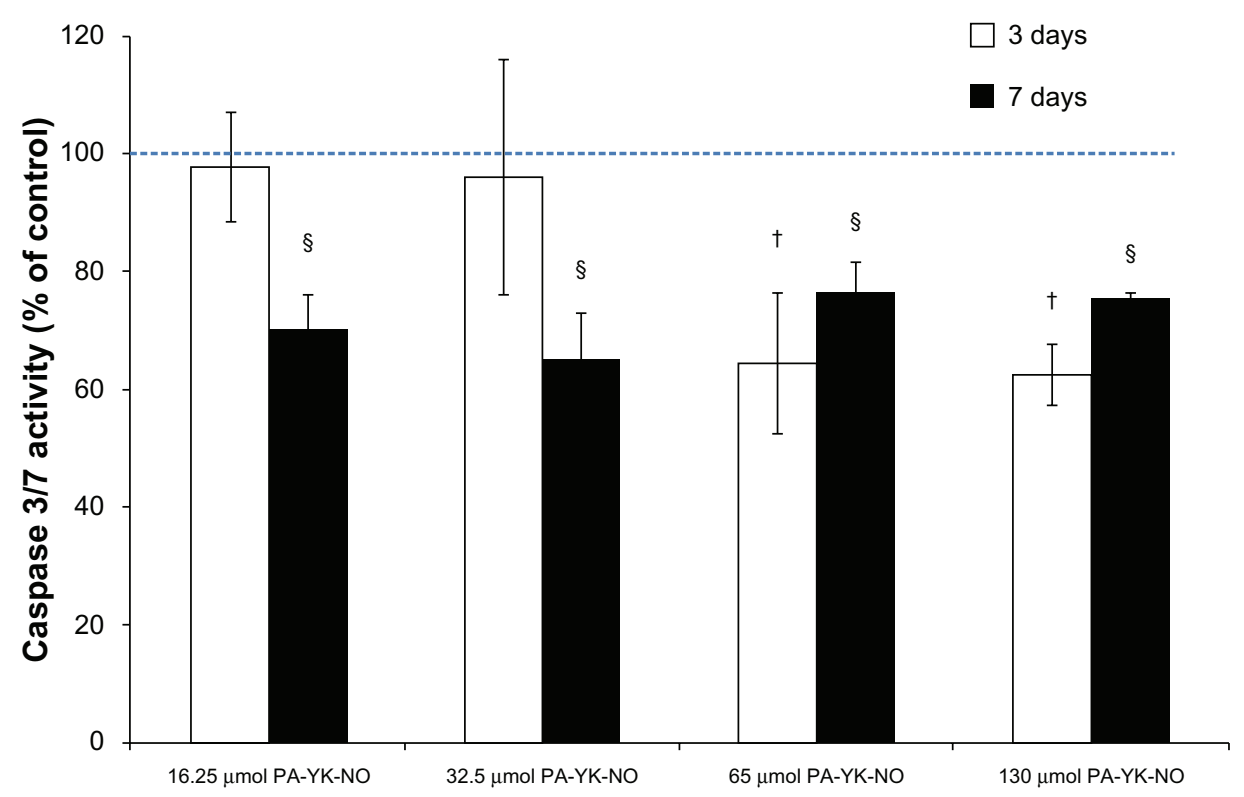

Figure 9 Effect of the nitric oxide-releasing peptide amphiphile nanomatrices on apoptosis in MIN-6 cells.

Notes: Each percentage of the caspase $3 / 7$ activity is compared with TCP serving as the control (dashed line). ${ }^{\dagger} P<0.05$ compared with day 3 control, $\$ P<0.05$ compared with day 7 control $(n=3)$.

Abbreviations: PA-YK-NO, nitric oxide-releasing peptide amphiphile nanomatrix; TCP, tissue culture plate. 
can release NO in a sustained manner over time, while promoting cell adhesion onto the extracellular matrix mimetic surface of the PA-YK-NO. This mechanism potentially has a dual effect as indicated by the fact the nanofibrous matrix could be utilized to both enhance islet functionality and improve islet engraftment by promoting NO-mediated angiogenesis post transplantation, thus enhancing islet survival. ${ }^{35,36} \mathrm{NO}$ is responsible for increasing endothelial cell proliferation, inhibiting apoptosis, and stimulating endothelial cell migration, thereby inducing the revascularization essential for successful islet transplantation. ${ }^{37}$

\section{Conclusion}

Based on recent findings regarding the beneficial role of NO, the aim of this study was to investigate the effect of biomimetic NO donors, which not only provide the native beta cell microenvironment through laminin-1 mimetic peptides but also release NO in a controlled manner over time. Nitric oxide-releasing PA nanomatrices showed a beneficial effect on glucose-stimulated insulin secretion, implying that this NO-producing scaffold could be utilized for improving pancreatic islet transplantation. Specifically, this study shows that there is a specific range of NO concentration that has a beneficial effect (Figure 8). Creating a biomimetic NOreleasing scaffold that releases NO continuously within a physiologically acceptable concentration range resulted in an improved response to glucose in MIN-6 beta cells. It was demonstrated that sustained release of NO by PA-YK-NO between $32.5 \mu \mathrm{mol}$ and $65 \mu \mathrm{mol}$ upregulates both PDX-1 and insulin gene expression, leading to improved functional responses of MIN-6 cells to glucose ( $22 \mathrm{mM}$ ). These findings are thought to be due to sustained release of NO because a similar level of apoptosis was observed between PA-YK-NOs, implying that the cell viability and survival rate were comparable with that of the control (Figure 9). In conclusion, sustained NO release at low concentrations from NO-releasing PA nanomatrices increases MIN-6 functionality, implying that NO has a multifaceted concentration-dependent effect on beta cells. Thus, the NO-releasing PA nanomatrix is a potential biomaterial for pancreatic islet transplantation, which is presently limited by technical problems such as angiogenesis and cell death.

\section{Acknowledgments}

The authors are grateful to Guanlan $\mathrm{Xu}$, who helped to operate the Prism 7000sequence detection system, and to Eun-Ha Lim, a scientific graphic designer who assisted with the graphic summary of this study. The authors gratefully acknowledge the MIN-6 cell line provided by Jun-Ichi Miyazaki (Osaka
University Graduate School of Medicine, Osaka, Japan). We also would like to thank Donald F Steiner (Department of Biochemistry, University of Chicago, IL, USA) and Wanxing Cui for obtaining the MIN-6 cell line. This study was supported by a UAB Diabetes Research Training Center pilot grant (P60 DK-079626 to JK), grants from the American Diabetes Association (1-09-IN-23 to HWJ; 1-09-JF-33 and 1-12-BS-99 to JK), NIDDK (1DP3DK094346-01 to HWJ), and an NSF career award (CBET-0952974 to HWJ), NIH (1R03EB017344-01 to $\mathrm{HWJ}$ ), and funding from the American Heart Association (13GRNT17220057 to JK), the Wallace H Coulter Foundation (to HWJ), a Woogok postdoctoral scholarship (to DJL), and a Caroline P Ireland scholarship and American Heart Association predoctoral fellowship (both to AA).

\section{Disclosure}

The authors report no conflicts of interest in this work.

\section{References}

1. Shapiro AM, Lakey JR, Ryan EA, et al. Islet transplantation in seven patients with type 1 diabetes mellitus using a glucocorticoid-free immunosuppressive regimen. $N$ Engl J Med. 2000;343:230-238.

2. Robertson RP. Islet transplantation as a treatment for diabetes - a work in progress. N Engl J Med. 2004;350:694-705.

3. Ryan EA, Lakey JR, Rajotte RV, et al. Clinical outcomes and insulin secretion after islet transplantation with the Edmonton protocol. Diabetes. 2001;50:710-719.

4. Stendahl JC, Wang LJ, Chow LW, Kaufman DB, Stupp SI. Growth factor delivery from self-assembling nanofibers to facilitate islet transplantation. Transplantation. 2008;86:478-481.

5. Chow LW, Wang LJ, Kaufman DB, Stupp SI. Self-assembling nanostructures to deliver angiogenic factors to pancreatic islets. Biomaterials. 2010;31:6154-6161.

6. Lim DJ, Antipenko SV, Anderson JM, et al. Enhanced rat islet function and survival in vitro using a biomimetic self-assembled nanomatrix gel. Tissue Eng Part A. 2011;17:399-406.

7. Lim DJ, Antipenko SV, Andukuri A, Corbett JA, Jun HW. Biological sensitivity to self-assembled nanomatrix platforms depends on the phenotype of MIN-6 beta-cells. Micro Nano Lett. 2011;6:619-623.

8. Hartgerink JD, Beniash E, Stupp SI. Self-assembly and mineralization of peptide-amphiphile nanofibers. Science. 2001;294:1684-1688.

9. Jun HW, Yuwono V, Paramonov SE, Hartgerink JD. Enzyme-mediated degradation of peptide-amphiphile nanofiber networks. Adv Mater. 2005;17:2612-2617.

10. Hartgerink JD, Beniash E, Stupp SI. Peptide-amphiphile nanofibers: a versatile scaffold for the preparation of self-assembling materials. Proc Natl Acad Sci U S A. 2002;99:5133-5138.

11. Kushwaha M, Anderson JM, Bosworth CA, et al. A nitric oxide releasing, self assembled peptide amphiphile matrix that mimics native endothelium for coating implantable cardiovascular devices. Biomaterials. 2010;31:1502-1508.

12. Andukuri A, Kushwaha M, Tambralli A, et al. A hybrid biomimetic nanomatrix composed of electrospun polycaprolactone and bioactive peptide amphiphiles for cardiovascular implants. Acta Biomater. 2011;7:225-233.

13. Andukuri A, Minor WP, Kushwaha M, Anderson JM, Jun HW. Effect of endothelium mimicking self-assembled nanomatrices on cell adhesion and spreading of human endothelial cells and smooth muscle cells. Nanomedicine. 2010;6:289-297. 
14. Andukuri A, Sohn YD, Anakwenze CP, et al. Enhanced human endothelial progenitor cell adhesion and differentiation by a bioinspired multifunctional nanomatrix. Tissue Eng Part C Methods. 2013;19: 375-385.

15. Stendahl JC, Kaufman DB, Stupp SI. Extracellular matrix in pancreatic islets: relevance to scaffold design and transplantation. Cell Transplant. 2009; 18:1-12.

16. Anderson JM, Patterson JL, Vines JB, Javed A, Gilbert SR, Jun HW. Biphasic peptide amphiphile nanomatrix embedded with hydroxyapatite nanoparticles for stimulated osteoinductive response. ACS Nano. 2011;5:9463-9479.

17. Anderson JM, Vines JB, Patterson JL, Chen H, Javed A, Jun HW. Osteogenic differentiation of human mesenchymal stem cells synergistically enhanced by biomimetic peptide amphiphiles combined with conditioned medium. Acta Biomater. 2011;7:675-682.

18. Tambralli A, Blakeney B, Anderson J, et al. A hybrid biomimetic scaffold composed of electrospun polycaprolactone nanofibers and self-assembled peptide amphiphile nanofibers. Biofabrication. 2009;1:025001.

19. Anderson JM, Kushwaha M, Tambralli A, Bellis SL, Camata RP, Jun HW. Osteogenic differentiation of human mesenchymal stem cells directed by extracellular matrix-mimicking ligands in a biomimetic self-assembled peptide amphiphile nanomatrix. Biomacromolecules. 2009; 10:2935-2944.

20. Anderson JM, Andukuri A, Lim DJ, Jun HW. Modulating the gelation properties of self-assembling peptide amphiphiles. ACS Nano. 2009;3:3447-3454.

21. Miyazaki J, Araki K, Yamato E, et al. Establishment of a pancreatic beta cell line that retains glucose-inducible insulin secretion: special reference to expression of glucose transporter isoforms. Endocrinology. 1990;127:126-132.

22. Smukler SR, Tang L, Wheeler MB, Salapatek AM. Exogenous nitric oxide and endogenous glucose-stimulated beta-cell nitric oxide augment insulin release. Diabetes. 2002;51:3450-3460.

23. McDaniel ML, Corbett JA, Kwon G, Hill JR. A role for nitric oxide and other inflammatory mediators in cytokine-induced pancreatic beta-cell dysfunction and destruction. Adv Exp Med Biol. 1997;426:313-319.

24. Corbett JA, Wang JL, Sweetland MA, Lancaster JR Jr, McDaniel ML. Interleukin 1 beta induces the formation of nitric oxide by beta-cells purified from rodent islets of Langerhans. Evidence for the beta-cell as a source and site of action of nitric oxide. J Clin Invest. 1992;90: 2384-2391.
25. Corbett JA, McDaniel ML. Intraislet release of interleukin 1 inhibits beta cell function by inducing beta cell expression of inducible nitric oxide synthase. J Exp Med. 1995;181:559-568.

26. Arnush M, Heitmeier MR, Scarim AL, Marino MH, Manning PT, Corbett JA. IL-1 produced and released endogenously within human islets inhibits beta cell function. J Clin Invest. 1998;102:516-526.

27. Mandrup-Poulsen T. The role of interleukin-1 in the pathogenesis of IDDM. Diabetologia. 1996;39:1005-1029.

28. Stevens RB, Lokeh A, Ansite JD, Field MJ, Gores PF, Sutherland DE. Role of nitric oxide in the pathogenesis of early pancreatic islet dysfunction during rat and human intraportal islet transplantation. Transplant Proc. 1994;26:692.

29. Xenos ES, Stevens RB, Sutherland DE, et al. The role of nitric oxide in IL-1 beta-mediated dysfunction of rodent islets of Langerhans. Implications for the function of intrahepatic islet grafts. Transplantation. 1994;57:1208-1212.

30. Ding Y, Rana RS. Nitric oxide does not initiate but potentiates glucoseinduced insulin secretion in pancreatic beta-cells. Biochem Biophys Res Commun. 1998;251:699-703.

31. Laffranchi R, Gogvadze V, Richter C, Spinas GA. Nitric oxide (nitrogen monoxide, NO) stimulates insulin secretion by inducing calcium release from mitochondria. Biochem Biophys Res Commun. 1995;217: 584-591.

32. Tejedo JR, Ramirez R, Cahuana GM, Rincon P, Sobrino F, Bedoya FJ. Evidence for involvement of c-Src in the anti-apoptotic action of nitric oxide in serum-deprived RINm5F cells. Cell Signal. 2001;13: 809-817.

33. Campbell SC, Richardson H, Ferris WF, Butler CS, Macfarlane WM. Nitric oxide stimulates insulin gene transcription in pancreatic betacells. Biochem Biophys Res Commun. 2007;353:1011-1016.

34. Kitiphongspattana K, Khan TA, Ishii-Schrade K, Roe MW, Philipson LH, Gaskins HR. Protective role for nitric oxide during the endoplasmic reticulum stress response in pancreatic beta-cells. Am J Physiol Endocrinol Metab. 2007;292:E1543-E1554.

35. Cooke JP, Losordo DW. Nitric oxide and angiogenesis. Circulation. 2002; 105:2133-2135.

36. Golocheikine A, Tiriveedhi V, Angaswamy N, Benshoff N, Sabarinathan R, Mohanakumar T. Cooperative signaling for angiogenesis and neovascularization by VEGF and HGF following islet transplantation. Transplantation. 2010;90:725-731.

37. Dimmeler S, Zeiher AM. Nitric oxide-an endothelial cell survival factor. Cell Death Differ. 1999;6:964-968.
International Journal of Nanomedicine

\section{Publish your work in this journal}

The International Journal of Nanomedicine is an international, peerreviewed journal focusing on the application of nanotechnology in diagnostics, therapeutics, and drug delivery systems throughout the biomedical field. This journal is indexed on PubMed Central,

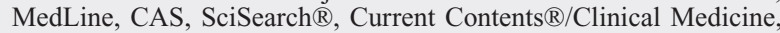

\section{Dovepress}

Journal Citation Reports/Science Edition, EMBase, Scopus and the Elsevier Bibliographic databases. The manuscript management system is completely online and includes a very quick and fair peer-review system, which is all easy to use. Visit http://www.dovepress.com/ testimonials.php to read real quotes from published authors. 